\title{
Pelvic Exenteration
}

National Cancer Institute

\section{Source}

National Cancer Institute. Pelvic Exenteration. NCI Thesaurus. Code C91837.

A major surgical procedure involving the removal of all the contents of the pelvic cavity, including reproductive organs, bladder, rectum, and anus. It is performed for cancers of the pelvic cavity organs that have not responded to other treatment options. 